\title{
Un siglo de cine político mexicano
}

institucional.us.es /ambitos/

José Ramón Santillán Buelna

Universidad Rey Juan Carlos

joseramon.santillan@urjc.es

English Version: A century of Mexican political cinema.

\section{Resumen}

Este artículo presenta el análisis de todas las películas mexicanas de temática política producidas durante los años de 1910 a 2014. La metodología utilizada consiste en un análisis de contenido de 54 largometrajes para identificar: año de producción, director, género, período y temática política que aborda, y también los premios nacionales e internacionales que recibieron. Los resultados muestran que

México cuenta con una importante filmografía y directores que han abordado acontecimientos claves de las transformaciones políticas del país en el último siglo.

\section{Palabras clave}

Cine político, México, régimen autoritario, cineastas, política.

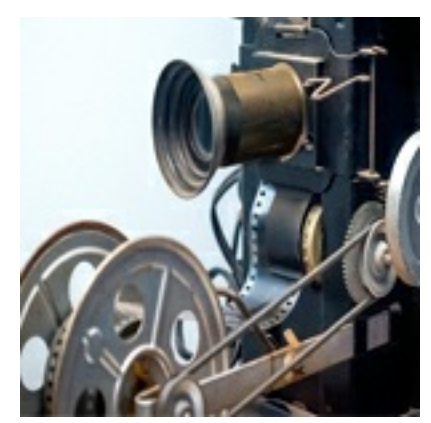

\begin{abstract}
This paper analyzes the mexican political filmes from 1910 to 2014. The paper studies 54 movies using the a content analysis to determine: production year, filmaker, film genre, political period, theme, and national and international awards won. The conclusions are Mexico has an important films and filmmakers that have addressed key events of political changes in the country in the last century.
\end{abstract}

Keywords

Mexican cinema, Mexico, authoritarian regime, filmmakers, mexican politics.

\section{INTRODUCCIÓN}

Las relaciones entre cine y política han producido una serie de estudios en el campo de la investigación en la comunicación de masas. Desde la aparición del cinematógrafo en París en el año de 1895 y su uso significativo como herramienta de propaganda política en los años 30 del

ÁMBITOS

2016

$n=33$ siglo pasado fue y sigue siendo un medio para conocer su impacto en las sociedades contemporáneas, como factor de información, difusión y adoctrinamiento político. Esta idea resulta igualmente válida para México, país en donde el cine llegó en 1886 se popularizó en la primera década del siglo pasado y fue creciendo paulatinamente a pesar de la intervención del Gobierno de Porfirio Díaz a través de un reglamento censurarlo por considerarlo "un espectáculo vulgar" (Moreno y Vázquez, 1983:9). Sin embargo siguió siendo un medio para retratar acontecimientos oficiales. Pero la Revolución Mexicana de 1910, el primer suceso político importante del siglo, fue llevado a la pantalla y marcó el inició del nexo entre la política y el cine mexicano alcanzando una relevancia como instrumento para registrar la dinámica social del país hasta nuestros días.

Está situación de la cinematografía como espacio para conocer la escenificación mediática de la política ha incentivado en la última década la atención de los investigadores en comunicación para conocer las influencias que puede tener como fuente de conocimiento y valoración de los actores y acontecimientos políticos. Para algunos investigadores está tendencia esta teniendo mayor interés en la ficción televisiva por el auge de series norteamericanas de éxito como El Ala Oeste de la Casa Blanca (Rodríguez-Virgili, J; Sádaba, y López-Hermida, A. 2010). Estos mismos autores mencionan que los estudios clásicos de la unión entre política y cine pueden agruparse en tres aspectos: la de recopilar e historiar las películas de tipo político, la relación entre la política y 
la industria especialmente de Hollywood; y el cine como compromiso o cine de protesta como arma política. Con estos antecedentes no sorprende que ahora los especialistas de la comunicación política en particular se vayan sumando a los sociólogos, antropólogos, historiadores, politólogos que habían hecho del cine su objeto de estudio: más cuando la centralidad comunicativa de la políticase ha convertido en una característica clave del siglo XXI. La idea es indagar en la representación fílmica de acontecimientos públicos y el funcionamiento del sistema político importante, una importante relación para construir una percepción de la política y de los políticos en los ciudadanos.

Como resultado de esa preocupación están apareciendo trabajos centrados en temáticas y efectos de películas y series de televisión de corte político. Los análisis van desde su función pedagógica hasta las representaciones que se hace de los asuntos públicos y de los políticos, pasando por aspectos de contenido e impacto en la audiencia, y la formación de opinión pública. En este aspecto los estadounidenses llevan la delantera en estudios de estos nuevos enfoques en el campo de la comunicación política. Ejemplo de ello es el liderazgo de sus investigadores y revistas académicas como Political Communication and Media Culture Society quienes dan un amplio abanico de abordajes teóricos y empíricos de esta cuestión.

Entre algunos de los estudios centrados en la personalización de la política, teniendo al presidente de los Estados Unidos como objeto de estudio en la ficción están los de Maarek, 1997; Negrine, Mancini y HoltzBacha, 2007. Otros trabajos recientes sobre la imagen del presidente en películas como Nixon (Oliver Stone, 1995), Air Force One (Wolfgang Petersen, 1997). En la primera se caracteriza al presidente desde una perspectiva humana para vincular con la audiencia, y en el segundo como un hombre valiente capaz de defender al país y a su familia (Crawley, 2006). Sin olvidar trabajos sobre irregularidades de la actuación del presidente y el papel de los periodistas para descubrirlas como el caso de Todos los hombres del presidente (Alan J. Pakula, 1973) o el de La cortina de humo (Barry Levinson, 1997) que trata de estrategias gubernamentales para distraer a la opinión pública fabricando una guerra. El análisis de estas cintas muestra el uso de la ficción para personificar el presidente y sus actuaciones donde se le atribuye influencia a la imagen y narrativa cinematográfica para representar determinados acontecimientos que tienen efectos políticos, como valorar al presidente en términos profesionales, éticos y humanos.

En México hay una gran tradición investigadora por parte de historiadores y escritores que han formado un sólido registro de la cinematografía nacional. Sobresale el nombre de Emilio García Riera, quien con su trabajo Historia documental del cine mexicano (1992, 1997), que consta de 18 tomos, y contiene las fichas completas y comentarios de todas las películas producidas entre 1929-1976, es una obra de referencia para estudiosos e investigadores. Está también el profesor Eduardo Vega Alfaro, quien junto con Leonardo García Tsao, crítico cinematográfico, desarrollan la Historia de la producción cinematográfica mexicana, 19772010, continuación de la obrade Emilio García Riera. Otro estudioso del cine mexicano es el escritor y crítico cultural Carlos Monsiváis (1997), quien dedicó varios trabajos al cine y su relación con la cultura popular, un trabajo en coautoría con Carlos Bonfil (1994) sobre la filmografía mexicana y su público. Estás son algunas obras para dar una idea de los trabajos y sus principales abordajes desde la pantalla.

En cuanto a la temática específica del cine con su relación política el acervo es escaso y a penas ha sido explorada. Encontramos trabajos puntuales, como el de Ruiz (2009) sobre el cine documental en la dinámica sociopolítica, analiza dos documentales del cineasta mexicano Luis Mandoki: ¿Quién es el Sr., López? y Fraude, México 2006, para reflexionar sobre la crítica al poder. También esta el trabajo de Jorge Porras (2008) quien hace un estudio comparativo entre México y España examinando el cine en un contexto de cambio político, otro texto en la misma línea es el de Salvador Velazco (2005) quien analiza las películas Rojo amanecer (Jorge Fons, 1989) y La ley de Herodes (Luis Estrada, 1999) como ejemplos de cine en tiempos de transición política.

En este marco de interés académico resulta importante seguir explorando esta temática en México con un sentido más amplio para profundizar en las relaciones entre política y cine. Se necesita primero realizar una clasificación de la producción cinematográfica de contenido político. Conocer cómo se ha abordado el tema en la ficción. Esas son algunas cuestiones que nos planteamos en el presente estudio. Nos interesa conocer el número de filmes en un período de más de 100 años, sus diferentes temáticas tratadas, el género cinematográfico... en suma, los datos básicos que formen una "radiografía" del cine político en México. Como un 
primer paso para después profundizar en sus contenidos e impacto político.

Partimos de la idea de que hay una larga tradición cinematográfica de temática política. Desde historias de la Revolución de 1910, de los setenta años de poder prísta 1929-1999, hasta la alternancia política del año 2000. También se debe considerar la censura oficial como un mecanismo del poder, hasta la década noventa del siglo pasado, para evitar películas que pudieran incomodar a los miembros del Gobierno en turno. Como ejemplo ilustrativo esta la cinta La sombra del Caudillo (Julio Bracho, 1960) que durante 30 años estuvo censurada porque se narra de forma critica la sucesión presidencial.

Creemos que algunos cineastas han dado cuenta de los cambios políticos y construido un importante acervo filmográfico para explicar y documentar temas, personajes e instituciones que forman parte de la historia política de México en el último siglo. Es por ello que la producción cinematográfica de este tipo de temática representa una oportunidad para conocer su papel como medio de comunicación en la representación de las transformaciones sociales del país. Se trata de indagar en un género poco estudiado, pero de gran relevancia porque es un espacio desde donde se construye la actividad pública y la función de los políticos.

Con esa finalidad diseñamos un análisis de contenido de las cintas políticas producidas durante los años de 1910 a 2014 (un período suficientemente amplio para identificar todas las producciones). La idea es hacer un "mapa" para tener la totalidad de películas filmadas durante esos años. El objetivo central es configurar el mosaico del cine político mexicano para posteriormente en otros trabajos conocer su función como fuente de propaganda, pedagogía, adoctrinamiento, registro histórico y recreación de la actividad política.

Objetivos del estudio.

1. Cuantificar las películas de temática política que se han producido durante los años de 1910-2014.

2. Identificar directores para saber quiénes las han filmado y si hay una regularidad de autores.

3. Clasificar el género cinematográfico desde el cual se ha abordado el acontecimiento político.

4. Conocer el período de la historia política que más se ha filmado.

5. Identificar qué tema político trata cada film.

6. Conocer la valoración artística de las películas por medio de los premios nacionales e internacionales que han recibido.

Hipótesis:

México cuenta con una importante filmografía política que ha abordado acontecimientos claves que dan testimonio de las transformaciones políticas del país en el último siglo.

\section{METODOLOGÍA}

Para analizar el cine político en México durante los años 1910-2014, primero delimitamos el marco temporal de estudio en tres períodos: 1910-1930 etapa de la Revolución; 1931-1987 el régimen autoritario, y 1988-2014 el proceso de democratización. Esta decisión metodológica nos sirvió para seleccionar como muestra de estudio 54largometrajes. El criterio para elegirlas fue tomar como referencia las obras de los más importantes recopiladores e investigadores del cine nacional como Emilio García Riera (1986), Jorge Ayala Blanco $(1986,1994)$ y Moisés Viñas (1997). También examinamos los Anuarios Estadísticos del Cine Mexicano editado por el Instituto Mexicano del Cinematografía (IMCINE) para corroborar todas las películas y evitar omisiones de cintas para analizar. Con esa información hicimos una selección de 54 películas políticas producidas entre los años de 1910 al 2014. Por temática política entendemos cualquier largometraje que recrea la realidad política en un film de ficción, que nos da mucha información del modo en qué se representa y escenifica la política, el poder y los políticos a lo largo del ciclo seleccionado.

Segundo, aplicamos una ficha de análisis de contenido a cada cinta con 10 variables de las cuales en esta ponencia presentamos seis que son 1) Identificar año de producción en períodos: a) 1910-1930 Etapa 
Revolución; b) 1931- 1987 Régimen autoritario; c) 1988-2014 Proceso de democratización; 2) director y cantidad de películas dirigidas en ese ciclo. 3) Género: a) Drama; b) Comedia; c) Documental; d) Intriga/Triller; e) Romance; f) Histórico; 4) Período que aborda: a) 1910-1930 Etapa Revolución; b) 1931- 1987 Régimen autoritario; c). 1988-2014 Proceso de democratización. 5). Clasificación del tema político concreto que trata en cuatro categorías: a) represión gubernamental, b) corrupción; c) político como personaje, d) funcionamiento del sistema político; y por último, 6) Si recibieron premios nacionales o internacionales, o ambos.

Tercero, analizamos la información utilizando el programa estadísticos SPSS para describir cantidad y ciclos temporales de la producción fílmica, determinar el tipo de género cinematográfico, temas y premios recibidos. Con esa información ofrecemos una clasificación y evolución histórica del cine político en México y su relación con los acontecimientos sociales del país dentro del período de 1910 al 2014 . Este estudio es un primer paso para investigar más adelante contenidos, protagonistas, recursos artísticos del cine político dentro de su papel como medio influyente en el proceso de comunicación política en nuestra sociedad.

Por lo tanto, las 54 películas que forman el corpus de nuestro análisis y que están ordenadas por año de producción y nombre del director son las siguientes:

1. El compadre Mendoza, 1933Fernando de Fuentes

3. Los de abajo, 1939Chano Ureta

5. Si yo fuera diputado. 1952Miguel M. Delgado

7. La sombra del Caudillo, 1956Julio Bracho

9. Reed, México Insurgente, 1973Paul Leducc

11. Calzonzin, InspectorAlfonso Arau

13. Canoa, 1975Felipe Cazals

15. El Pando, 1975Felipe Cazals

17. Cuartelazo, 1976Alberto Isacc

19. El año de la peste, 1978Felipe Cazals

21. Bajo la metralla, 1982Felipe Cazals

23. Morir en el Golfo, 1988Alejandro Pelayo

\section{Rojo Amanecer, 1989Jorge Fons}

27. El extensionista, 1991Juan Pérez Gavilán

29. Playa Azul, 1992Alfredo Joskowicz

31. Maten al candidato, 1998Giberto y Adolfo Martínez

33. Pachito Rex, me voy pero no del todo, 2001, Fabián Hofman

35. Conejo en la luna, 2004Jorge Ramírez

37. Un Mundo maravilloso, 2006Luis Estrada

39. Cementerio de papel, 2008Mario Hernández
2. Vámonos con Pancho Villa, 1935Fernando de Fuentes

4. Viva Zapata, 1952Elia Kazan

6. El impostor, 1956Emilio Fernández

8. Distinto amanecer, 1968Julio Bracho

10. Ante el cadáver de un líder, 1973Alejandro Galindo

12. Las fuerzas vivas, 1975Luis Alcoriza

14. Maten al Léon, 1975Luis Estrada

16. Las poquianchis, 1976 Felipe Cazals

18. Bandera Rota, 1978Gabriel Retes

20. Cadena perpetua, 1979Arturo Risptein

22. Días difíciles, 1987Alejandro Pelayo

24. ¿Nos traicionará el presidente?, 1988Juan Pérez Gavilán

26. Político por error, 1991Alejandro Todd

28. El Bulto, 1991Gabriel Retes

30, Fibra óptica, 1997Francisco Athié

32. La ley de Herodes, 1999Luis Estrada

34. Magnicidio, complot en Lomas Taurinas, 2002, Miguel Marte

36. El Violín, 2005Francisco Vargas

38. Arráncame la vida, 2008Roberto Sneider

40. Corazón del tiempo, 2009Alberto Cortés 
41. Chico grande, 2009Felipe Cazals

43. Borra de la memoria, 2010Alfredo Gurrola

45. El atentado, 2010Jorge Fons

47. La cebra, 2011Fernando Javier León

49. El lenguaje de los machetes, 2011Kyzza Terrazas

51. Cristiada, 2012.Dean Wright

53. La dictadura perfecta, 2014Luis Estrada
42. El traspatio, 2009Carlos Carrera

44. El infierno, 2010Luis Estrada

46. Ella y el candidato, 2011Roberto Girauld

48, Revolución, 2010Varios directores

50. Colosio: el asesinato, 2012Carlos Bolado

52. Ciudadano Buelna, 2013Felipe Cazals

54. Tlatelolco, verano del 68, 2014Carlos Bolado

\section{ANÁLISIS}

\subsection{Número de películas de temática política}

El número total de películas de temática política que integran el estudio muestran que se produjeron un total de 54 en los tres periodos investigados. Para los años 1910-1930 dos; en 1931-1987 son 20 y para 1987-2014 fueron 32 (tabla 1). Los datos permiten observar que la mayor creación se dio en el tercer período, 59,3\% de las cintas, que pone de manifiesto un mayor interés de los cineastas en filmar asuntos políticos de la dinámica social del país. Seguidamente, se sitúan los años de 1931-1987 con el 37\% de los filmes de tipo político. Por último, en los años de 1910 al 1930 sólo se identificaron dos películas relativas al tema de estudio.

Tabla 1. Número de peliculas politicas producidas durante los años de 1910-2014

\begin{tabular}{|c|c|c|}
\hline Periodo de producción & No. de peliculas & $\%$ \\
\hline $1910-1930$ & 2 & 3,7 \\
\hline $1931-1987$ & 20 & 37,0 \\
\hline $1987-2014$ & 32 & 59.3 \\
\hline Total & 54 & 100 \\
\hline
\end{tabular}

Profundizando en los directores que han filmado algunas de estas 54 cintas para conocer si existen cineastas representativos del cine político en México que han mantenido una continuidad, tenemos que sobresalen Felipe Cazals con siete (Canoa,1975, El Apando,1975, Las Poquianchis, 1976, El año de la peste, 1978, Bajo la metralla, 1982, Chico grande, 2009, Ciudadano Buelna, 2013). Después le sigue Luis Estrada con cinco (Maten al León,1975, La ley de Herodes, 1999; Un mundo maravilloso, 2006, El infierno, 2010; La dictadura perfecta, 2014). Sobresalen siete directores con dos películas cada uno: Fernando de Fuentes con El compadre Mendoza, 1933 y Vámonos con Pancho Villa, 1935; Julio Bracho es el director de La sombra del caudillo, 1960 y de Distinto amanecer, 1968; Gabriel Retes filmó Bandera Rota, 1978 y El Bulto, 1991; Juan Pérez Gavilán con Nos traicionará el presidente, 1988; El extensionista, 1991; Alejandro Pelayo es el realizador de Días difíciles, 1987 y Morir en el Golfo, 1988; Jorge Fons es el autor de Rojo amanecer; 1989 y El atentado, 2010; y por último, Carlos Bolado con Colosio: el asesinato, 2012 y Tlatelolco, verano del '68, 2014.

En primer aspecto con estos datos podemos explicar que dos directores Felipe Cazals y Luis Estrada han filmado 12 películas de las 54 contabilizadas. Los dos aportan el 22,22\% del total, su importancia numérica les concede gran protagonismo dentro de la historia del cine político mexicano. La buena valoración de sus trabajos por el público y la crítica reafirma su papel como realizadores que han sabido dar testimonio del poder, las luchas sociales y los conflictos internos relacionados con el ejercicio del poder político.

El segundo aspecto, sobresalen siete directores con dos películas cada uno: Fernando de Fuentes, Julio Bracho, Gabriel Retes, Juan Pérez Gavilán, Alejandro Pelayo, Jorge Fons y Carlos Bolado, han filmado 14 cintas y cuantifican el $26 \%$ de la producción total. Pese a situarse por debajo de la cantidad de cintas personales dirigidas por Felipe Cazals y Luis Estrada, son referentes en el cine político con sus películas, que son de alta calidad argumentativa y técnica, ya se encuentran dentro de la historia del cine político en México. 


\subsection{Género}

Junto al volumen de cintas producidas y sus autores, otro dato importante para conocer la relevancia del cine político en México es el tipo de género en que se abordó dicha cuestión, los datos aparecen el la tabla 2. En el período temporal analizado sobresale el drama en 30 cintas, con un 55,6\% más de la mitad del total. Como segundo dato relevante es la comedia en 15 filmes. Después sigue el suspenso/ thriller con ocho, y por último sólo en una película se toca la política en clave de romance.

Tabla 2. Género

\begin{tabular}{|c|c|c|}
\hline Gónero & Frecuencia & $\%$ \\
\hline Drama & 30 & 55,6 \\
\hline Comedia & 15 & 27,8 \\
\hline Suspenso/Thriller & 8 & 14,8 \\
\hline Romance & 1 & 1,9 \\
\hline Total & 54 & 100 \\
\hline
\end{tabular}

Fuente: Elaboración propia

El análisis del género cinematográfico como técnica argumental del cine político muestra que el drama fue el más utilizado. Tratar las temáticas políticas desde una perspectiva más formal con intenciones de inspirar seriedad y dar testimonio para interpretar problemas del ejercicio de la política y las consecuencias de las decisiones que se toman se convirtió en el punto de más vista más utilizado por los cineastas. El segundo género utilizado fue la comedia aquí se trató de provocar risa o gracia sobre las cuestiones públicas tratadas y de los políticos como principales protagonistas. El suspenso o thriller fue adoptado como tercer recurso cinematográfico cuyo objetivo era mantener al espectador en estado de tensión en cuanto el desarrollo de las historias políticas que trataban las cintas.

\subsection{Período político abordado en las películas}

Tabla 3. Periodo politico que abordan las peliculas

\begin{tabular}{|l|l|l|}
\hline \multicolumn{1}{|c|}{ Periodo politico } & Frecuencia & Porcentaje \\
\hline 1910-1930 Revolución & 11 & 20,4 \\
\hline 1931-1987 Régimen autoritario & 26 & 48,1 \\
\hline $\begin{array}{l}\text { 1988-2014 Proceso de } \\
\text { democratización }\end{array}$ & 17 & 31,5 \\
\hline Total & 54 & 100 \\
\hline
\end{tabular}

Fuente: Elaboración propia

La época política que más se ha filmado en México es el período autoritario, entendido como la preeminencia del PRI y del presidencialismo que desde la fundación del partido en 1929 habían el control todas las instituciones del país hasta la apertura democrática que inició en 1988. Este ciclo fue el que más interesó a los cineastas para abordar las situaciones que representaba un gobierno dominante que amenazaba, censuraba o reprimía a las personas y opiniones que cuestionaban el status quo. En la fase democratizadora que va de 1988 al 2014 se filmaron 17 cintas, esta fase temporal se define como un modelo de transición del autoritarismo a una apertura política. También se identifica con el gobierno y su partido el PRI en crisis, el diseño de un sistema de partidos plural, elecciones equitativas. Con espacios de debate y la inclusión de la sociedad en los temas de discusión pública. En este contexto es donde se da le segundo mayor registro de temáticas políticas llevadas al cine. Por último, el período revolucionario (1910-1930) fue el tercer momento político que recibió la atención de los cineastas con once cintas. Este momento ha sido muy recreado sobre todo con una visión crítica de la lucha revolucionaria y sus líderes.

\subsection{Temas de las películas}

En cuanto a los temas políticos que tratan las películas se detecta un claro predomino del sistema político con 17 filmes, el $31,5 \%$ del total. Se trata de películas centradas en cuestiones del funcionamiento de la política como la gestión institucional; en el ámbito judicial y la aplicación de leyes; al PRI y la lucha interna por el poder político desde la sucesión presidencial hasta el asesinato político. En los filmes analizados sobre los déficits de los sistemas de procuración e impartición de justicia, y, en general de las condiciones del Estado de derecho en 
México sobresalen Bajo la metralla, el año de la peste, El apando de Felipe Cazals; Cadena perpetua, Arturo Ripstein; Con respecto a interés y causas relacionadas con la lucha por el poder político están La sombra del caudillo, Julio Bracho; Magnicidio, Complot en Lomas Taurinas, Miguel Marte; Y con el funcionamiento del sistema político están La Ley de Herodes y La dictadura perfecta de Luis Estrada, entre otras.

En segundo lugar, con un 25,9\%, se encuentra la temática referidas a la represión gubernamental. En las primeras se engloban aquellas relacionadas con acontecimientos como La matanza de estudiantes del 2 de octubre de 1968 (Rojo amanecer, Jorge Fons, Borrar de la memoria, Alfredo Gurrola; Tlatelolco verano del '68 de Carlos Bolado) o la represión del Jueves de Corpus del 10 de junio de 1971, donde fueron reprimidos los estudiantes por órdenes del Gobierno, con películas como El Bulto, Gabriel Retes. También El Violín de Francisco Vargas sobre la guerra sucia de los años setenta. Como segundo aspecto de represión institucionalizada de políticos, caciques, empresarios y diversos cuerpos policiales involucrados hay filmes como Bandera Rota de Gabriel Retes; El Extensionista de Juan Pérez Gavilán; El lenguaje de los machetes de Kyzza Terrazas;

En tercer y cuarto lugar, aparecen dos temas la corrupción y los políticos como personajes con 2l cintas el $20,4 \%$ cada uno. Los primeros filmes se focalizan en actuaciones ilegales de políticos para abusar de su poder y enriquecerse, o actuar coludidos con otros actores sociales como caciques, autoridades locales, policías para sacar provecho. En esta temática están las cintas Compadre Mendoza, Fernando de Fuentes, Las Poquianchis, Felipe Cazals; Playa Azul de Alfredo Joskowicz; Conejo en La Luna, Jorge Ramírez; Un mundo maravilloso de Luis Estrada; Cementerio de papel, Mario Hernández; El traspatio, Carlos Carrera;

Tabla 4. Temáticas de las peliculas

\begin{tabular}{|l|l|l|}
\hline Temas & Frecuencias & $\%$ \\
\hline Sistema politico & 17 & 31,5 \\
\hline Represión gubernamental & 14 & 25,9 \\
\hline Corrupción & 11 & 20,4 \\
\hline Políticos como personajes & 11 & 20,4 \\
\hline Otros & 1 & 1,9 \\
\hline Total & 54 & $\mathbf{1 0 0}$ \\
\hline
\end{tabular}

Fuente: Elaboración propia.

En cuanto, a los políticos como personajes comprende las películas que tratan a los miembros del sistema político en clave de parodia su vida, trayectoria o conflictos personales y familiares, o para desmitificar su papel en la historia del país. Están los filmes: Vámonos con Pancho Villa de Fernando de Fuentes; Viva Zapata de Elia Kazan, Si yo fuera Diputado de Miguel Delgado; Ante el cadáver de un líder de Alejandro Galindo; Las fuerzas vivas de Luis Alcoriza; Calzonzin inspector de Alfonso Arau; Morir en el Golfo de Alejandro Pelayo; Arráncame la vida de Roberto Sneider; Ella y el candidato de Roberto Girauld; Ciudadano Buelna de Felipe Cazals.

Las cuatro referencias temáticas que hemos descrito forman parte de un análisis que muestra en un sentido amplio el funcionamiento del sistema político y las actuaciones de sus protagonistas que contribuyen a esbozar una metodología para profundizar en el estudio de los contenidos políticos en las películas.

\subsection{Premios nacionales e internacionales}

En este apartado examinamos los reconocimientos tanto nacionales como internacionales que han recibido las películas de temática política. Tenemos que 40 de las 54 han recibido algún galardón, es decir, el $74 \%$ de las cintas analizadas. Este dato nos permite observar una importante calidad artística y técnica en los filmes políticos que han sido llevados a la pantalla como muestra el Gráfico 1. 


\section{Gráfico 1. Peliculas premiadas a nivel nacional e internacional}

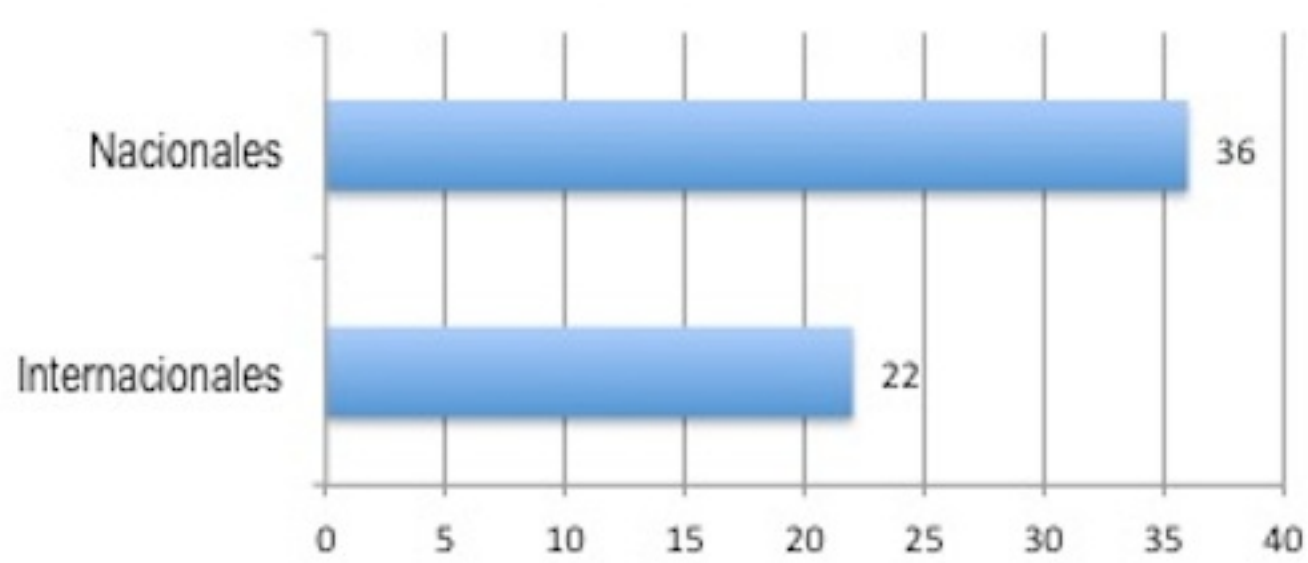

En primer lugar, 36 películas han recibido el Ariel principal premio nacional que entrega cada año desde 1947 la Academia Mexicana de Artes y Ciencias en reconocimiento a los trabajos cinematográficos. En cuanto a premios internacionales se contabilizan 22 de las 54 películas homenajeadas en el extranjero en Festivales como el Internacional de Cine de Karlovi, Festival Internacional de Nuevo Cine Iberoamericana, Festival de Huelva, Festival de Cine de Valladolid, entre otros. Con estos premios cintas y directores han sido reconocidos en el mundo por su talento para contar asuntos políticos de acontecimientos, personajes e instituciones del país. Las 40 películas premiadas tanto a nivel nacional como internacional confirman que en México existe una filmografía política de calidad que plantea profundizar en el análisis de los filmes como testimonios de las transformaciones políticas ocurridas durante todo un siglo. Una etapa que comprende desde la Revolución en 1910 pasando por el régimen autoritario de los años 1931-1987 hasta el proceso de democratización que inició en 1988,que tuvo un momento culmen en el 2000 con el primer presidente de oposición que impulsó la transformación política del país.

\section{CONCLUSIONES}

En este trabajo hemos analizado el cine político en México durante los años de 1910 al 2014. Identificamos y clasificamos 54 películas de ficción que mantienen una estrecha relación con la temática política. Destacada la producción fílmica en las tres últimas décadas (1988-2014). Sobresale la filmación de acontecimientos como la Matanza de estudiantes en 1968, la ineficiencia del gobierno, la sucesión presidencial, la corrupción del sistema político (PRI), y de momentos claves de los años recientes, década de los noventa, que iniciaron el proceso democratización: elecciones presidenciales de 1988, rebelión zapatista de 1994, asesinato del candidato presidencial Colosio 1994, entre otros. En la mayoría de las películas de ficción que se han estudiado la temática aparece narrada de forma indirecta en tres géneros cinematográficos (drama, comedia, suspenso o thriller).

Por otra parte, encontramos dos cineastas Felipe Cazals y Luis Estrada que se erigen como representantes del cine político mexicano por los temas de sus trabajos que se vinculan muy estrechamente con el funcionamiento del sistema político, la figura de los políticos y del PRI. La calidad de sus películas han recibido el reconocimiento tanto nacional como internacional. También observamos cintas y directores que forman parte de la historia del cine mexicano como: El compadre Mendoza, Fernando de Fuentes; La sombra del caudillo, Julio Bracho; Rojo Amanecer, Jorge Fons, o la aparición de nuevos temas y realizadores como: Colosio: el asesinato, Carlos Bolado que va del crimen del candidato del PRI en 1994, o El lenguaje de los machetes de Kyzzya Terrazas, que trata de los disturbios de Salvador Atenco en 2006 entre policías y ciudadanos, o Corazón del tiempo de Alberto Cortés que habla de la Rebelión del Ejercito Zapatista de Liberación Nacional (EZLN). Con estos filmes se sigue construyendo el campo del cine político en México un género con gran tradición desde hace 100 años, como hemos visto en este estudio. 
Por último, los resultados de esta investigación servirán para seguir profundizando en los contenidos políticos en las que se centran los filmes, la relación entre gobernantes y ciudadanos, y la percepción de la política y de los políticos que se construye desde el cine.

\section{BIBLIOGRAFÍA}

ANUARIO ESTADISTICO DEL CINE MEXICANO (2015): Instituto Mexicano de Cinematografía. México.

AYALA, J. (1986): La búsqueda del cine mexicano. UNAM, México, 1974 [2a ed. Posada, 1986]. (1994): La eficacia del cine mexicano.Grijalbo, México,

CRAWLE, J. (2006): Mr. Sorkin Goes to Washington, Shaping the Presiden ton Television's The West Wing. North Carolina, MacFarland.

DE LA VEGA, E. y GARCIA TSAO, L. (2011) : Historia de la producción cinematográfica mexicana, 19772010. Universidad de Guadalajara. México.

GARCIA RIERA, E. (1992-1997): Historia Documental del cine mexicano. 18 tomos. Universidad de Guadalajara, Consejo Nacional para la Cultura y las Artes (CONACULTA), Secretaría de Cultura del Gobierno del Estado de Jalisco y el Instituto Mexicano de Cinematografía (IMCINE)

(1998):Breve historia del cine mexicano. Primer Siglo 1897-1997, México, Conaculta. Imcine/ Ediciones Mapa/Canal 22/ Universidad de Guadalajara,.

MONSIVAIS, C. (1997): Rostros del cine mexicano. México: Américo Artes Editores S.A de C.V., PORRAS, J. (2008): "El cine como instrumento de reinterpretación histórica en periodos de transición democrática”. Ciencias Sociales . Vol. IV. No. 122 pp. 89-101

MONSIVAIS, C. y BONFIL, C. (1994): A través del espejo: el cine mexicano y su público. México: Ediciones El Milagro; Instituto Mexicano de Cinematografía,

MAAREK, P. (1997): Marketing político y comunicación. Barcelona: Paidós.

MORENO BRIZUELA, D. y VÁZQUEZ GOMEZ R. (1983) : “Políticas

Cinematográficas". UNAM: Escuela de Ciencias Sociales. México D.F

NEGRINE, MANCINI y HOLTZ-BACHA. (2007): The professionalization of Political Communication. Bristol: Intellect.

RODRIGUEZ VIRGILI, J; SÁBADA, T, LÓPEZ HERMIDA, A. (2010): "La ficción audiovisual como nuevo escenario para la Comunicación Política”. CIC Cuadernos de Información y Comunicación, vol. 15 37-54 pp.

RUIZ, A. (2009): "El desarrollo del cine y el videodocumental en la dinámica sociopolítica del México contemporáneo". En Claves del Pensamiento, vol. III, núm. 6, julio - diciembre, 2009, pp. 153-167 ITESMCiudad de México Distrito Federal, México.

VELAZCO, S. (2005): "Rojo amanecer y La ley de Herodes: cine político de la transición política mexicana". Hispanic Research Journal: Iberian and Latin American Studies. Vo. 6, Issue 1. pp. 67-80.

VIÑAS, M. (1987).: Historia del cine mexicano. UNAM-UNESCO. México. 


\section{BREVE SEMBLANZA DEL AUTOR}

José Ramón Santillán Buelna es Doctor en Ciencia Política por la Universidad Pompeu Fabra. Desde 2006 es profesor Titular (i) en la Universidad Rey Juan Carlos (URJC) donde imparte las asignaturas de Comunicación Política y de Periodismo de Análisis y Opinión. También es docente en el Máster Oficial de Investigación Aplicada a la Comunicación y miembro del Grupo de Estudios Avanzados de la Comunicación (GEAC) de la misma institución. Ha escrito dos libros completos y cuatro capítulos sobre prensa, elecciones y democracia en México. Y doce artículos en revistas científicas como Observatorio (OBS), Zer, Andamios, Global Media Journal México, REDMARKA, Razón y Palabra en temas relacionados con la Comunicación y el Periodismo.

Ámbitos. Revista Internacional de Comunicación, n.33, edición de verano, 2016.

Recibido: 23/05/2016

Aprobado: 01/06/2016 